\title{
基于新型杜铃状双富勒烯衍生物受体材料的高开路电压 聚合物太阳能电池
}

\author{
郭颖*,a 朱华新 ${ }^{a}$ 刘桂林 ${ }^{a}$ 严慧敏 ${ }^{a}$ 朱冰洁 ${ }^{a}$ \\ 李 帅 ${ }^{a}$ 孙亚军 ${ }^{a}$ 李果华 $a, b$ \\ ( ${ }^{a}$ 江南大学理学院 无锡 214122) \\ ( ${ }^{b}$ 江苏省(尚德)光伏技术研究院 无锡 214028)
}

\begin{abstract}
摘要 设计合成一种具有杜铃状双富勒烯衍生物, 其分子结构采用 ${ }^{1} \mathrm{H} \mathrm{NMR},{ }^{13} \mathrm{C} N \mathrm{NR}$, 元素分析和 MS 进行了结构表 征, 并运用 Gaussian 量子化学程序对其分子的几何构型进行结构优化; 通过对其结构的设计, 有效地改变其在有机溶 剂中的溶解性, 将其与聚合物 P3HT 共混后制备太阳能电池, 当 P3HT 与其质量混合比为 $1: 0.5$ 时, 并在 $110{ }^{\circ} \mathrm{C}$ 下进 行退火处理, 其太阳能电池开路电压达到了 $0.89 \mathrm{~V}$, 光电转换效率为 $1.01 \%$, 短路电流为 $2.73 \mathrm{~mA} / \mathrm{cm}^{2}$, 填充因子 $\mathrm{FF}$ 为 $39.1 \%$. 此研究为获得高开路电压的聚合物太阳能电池提供了一种可行性选择.
\end{abstract}

关键词＼cjkstart杜铃状双富勒烯衍生物; 聚合物太阳能电池; 光电转换效率; 开路电压

\section{Polymer Solar Cells with High Open-Circuit Voltage Based on Novel Barbell-Shaped Bifullerene Derivative as Acceptor}

\author{
Guo, Ying ${ }^{*, a} \quad$ Zhu, Huaxin ${ }^{a} \quad$ Liu, Guilin $^{a} \quad$ Yan, Huimin $^{a} \quad$ Zhu, Bingjie ${ }^{a}$ \\ Li, Shuai ${ }^{a} \quad$ Sun, Yajun ${ }^{a} \quad$ Li, Guohua ${ }^{a, b}$ \\ ( ${ }^{a}$ School of Science, Jiangnan University, Wuxi 214122) \\ ( ${ }^{b}$ Jiangsu (Suntech) Institute for Photovoltaic Technology, Wuxi 214028)
}

\begin{abstract}
Polymer solar cells (PSCs) based on fullerene and its derivatives as acceptors are attracting extensive interest due to their advantages of wide materials, low cost and flexibility. The research of the accepting materials is considered to be a key factor to obtain high-efficiency PSCs, especially fullerene derivatives. In this regard, a novel barbell-shaped bifullerene derivative was designed and synthesized, and the structure was identified by ${ }^{1} \mathrm{H}$ NMR, ${ }^{13} \mathrm{C}$ NMR, MS and element analysis. Its absorption, electrochemistry, and geometry were studied systematically by UV, CV and Gaussian. Results showed that the bifullerene derivative can be used as acceptor for PSCs. The solubility of the derivative was effectively changed by increasing the alkyl chain in the molecule. The PSCs were fabricated with the structure of ITO/PEDOT:PSS/P3HT:bifullerene derivative/Ca/Al. After optimization of the ratio of bifullerene derivative and P3HT, the cells with the optimized conditions show that the power conversion efficiency (PCE) was $1.01 \%$ with $J_{\mathrm{sc}}$ of $2.73 \mathrm{~mA} / \mathrm{cm}^{2}$ and $V_{\mathrm{oc}}$ of $0.89 \mathrm{~V}$. It is noteworthy that the open-circuit voltage was up to $0.89 \mathrm{~V}$, which is more than $0.3 \mathrm{~V}$ that of $\mathrm{PC}_{60} \mathrm{BM}\left(V_{\mathrm{oc}}=0.56 \mathrm{~V}\right)$. Our result provides a feasible choice for PSCs with high open-circuit voltage.
\end{abstract}

Keywords barbell-shaped bifullerene derivative; polymer solar cells; power conversion efficiency; open-circuit voltage

基于富勒烯及其衍生物的聚合物太阳电池(PSC)因 其制备简单、成本低廉、重量轻及可制成柔性器件等优 点 ${ }^{[1 \sim 3]}$, 倍受国内外研究者的广泛关注, 成为太阳能电 池研究领域的重要研究方向. 富勒烯作为聚合物太阳能
电池受体材料的特性最早被 Sariciftci 等 ${ }^{[4]}$ 发现, 并成功 制备了基于聚对苯撑乙炔 $(\mathrm{PPV}) / \mathrm{C}_{60}$ 双层膜异质结太阳 能电池. 随后, 关于富勒烯及其衍生物作为太阳能电池 受体材料的研究发展迅猛, 相关报道层出不穷 ${ }^{[5 \sim 8]}$. 其

\footnotetext{
* E-mail: iamguoying@aliyun.com

Received June 11, 2015; revised August 19, 2015; published online September 15, 2015.

Project supported by the Fundamental Research Funds for the Central Universities (No. JUSRP111A22).

中央高校基本科研业务费(No. JUSRP111A22)资助项目.
} 
研究工作主要集中在对富勒烯进行功能化修饰, 有效地 调节富勒烯分子与聚合物半导体材料相容性, 从而使其 在器件中形成有利于电荷传输的网络互穿结构, 提高其 光电转换效率. Fréchet 等 ${ }^{[9]}$ 将含富电子甲氧基苯环以及 缺电子氟原子的苯环引入到富勒烯上, 制备了一系列可 溶性的富勒烯受体材料, 其与聚三己基噻吩(P3HT)制备 的太阳能电池的最高能量转化效率可达到 $4.5 \%$. 通过 外围取代基的修饰，可以有效地调节富勒烯衍生物与 P3HT 之间相容性, 从而提高太阳能电池的光电效率. 目前最常用的碳球类受体材料主要集中在 $\mathrm{PC}_{61} \mathrm{BM}$ 和 $\mathrm{PC}_{71} \mathrm{BM}$, 其优点是溶解性好和载流子迁移率适中, 与 P3HT 混合制备的 PSC 能量转化效率最高可达 $6.5 \%$ 左 右, 但其缺点是可见区吸收弱和 LUMO 能级过低, 从而 限制了其光电效率的提升. Blom 等 ${ }^{[10]}$ 发现通过对富勒 烯衍生物进行双取代, 可以不同程度地增强其在可见光 的吸收, 并明显提高富勒烯衍生物的 LUMO 能级, 因 而, 基于富勒烯多取代衍生物开始得到重视, 富勒烯双 加成衍生物也成为提高 PSC 效率的主要手段, 一些双加 成或多加成的富勒烯衍生物作为 PSC 的受体材料相继 出现 ${ }^{[11 ~ 14]}$. 如李永舫课题组采用给体一受体(D-A)反应 合成了两种狮取代结构的富勒烯衍生物狮-C60 单加成 物 ICMA 和新型茚双加成 C60 衍生物 $\mathrm{ICBA}^{[15]}$, 其中以 ICBA 为受体的电池开路电压达到 $0.8 \mathrm{~V}$, 光电转换效率 达到 $7 \%$. 然而富勒烯双或多加成衍生物普遍存在异构 体较多、难提纯、可重复性差等缺点, 严重影响电池器 件的重复性和稳定性, 另外, 随着富勒烯的取代基逐渐 增加, 其导电性能也急剧下降. 鉴于此, Wong ${ }^{[16]}$ 和 $\mathrm{TaO}^{[17]}$ 等相继报道了使用双富勒烯衍生物作为太阳电池 的受体材料, 研究结果发现此类型受体材料具有优越的 载流子传输性能, 其中以双富勒烯衍生物 FHPCBC 作 为单活性层的 PSC 电池, 开路电压达到了 $0.9 \mathrm{~V}$, 比传 统的富勒烯衍生物 PCBM 和新型狮双加成 C60 衍生物 ICBA 的开路电压均提高了 $0.1 \sim 0.3 \mathrm{~V}$ 左右. 然而, 关于 含双富勒烯衍生物的研究还相对较少, 因此, 设计合成 含双富勒烯的衍生物成为关键. 本论文通过设计制备一 种在单一分子中引入两个富勒烯结构单元, 其合成路线 见 Scheme 1. 在分子构建中利用芴的长烷基链增加其分 子的自由空间体积, 改变双富勒烯衍生物的溶解性, 并 系统的研究了其作为太阳能电池受体材料的光伏性能.

\section{1 结果与讨论}

\section{1 富勒烯衍生物的光学及电化学性能}

富勒烯衍生物 $\mathbf{5}$ 在甲苯溶液中的紫外-可见吸收光 谱图表明, 化合物 5 从 $725 \mathrm{~nm}$ 起一直到 $390 \mathrm{~nm}$ 都有不 同强度的吸收, 主要在 432 和 $708 \mathrm{~nm}$ 处 2 个明显的峰,
图 1(a)中插图是 $708 \mathrm{~nm}$ 处放大的峰型，与 $\mathrm{PC}_{61} \mathrm{BM}$ 的吸 收基本一致, 因此, 将化合物 $\mathbf{5}$ 作为聚合物太阳能电池 中受体材料, 与 $\mathrm{P} 3 \mathrm{HT}$ 能够形成有效的激子分离. 化合 物 5 的最低空轨道(LUMO) 是通过循环伏安法进行测定, 如图 1(b)所示, 其 LUMO 能级为 $-3.68 \mathrm{eV}$, 与 $\mathrm{PC}_{61} \mathrm{BM}$ 的 LUMO 能级比较 $(-3.91 \mathrm{eV})$ 发现, LUMO 能级提高了 约 $0.23 \mathrm{eV}$, 对应的还原峰归因于 $\mathrm{C}_{60}{ }^{-} / \mathbf{5}$, 第二对氧化还 原峰归因于 $\mathrm{C}_{60}{ }^{2-} / \mathbf{5}$. 根据本体异质结太阳能电池的开路 电压与其受体 LUMO 的关系: $V_{\mathrm{OC}} \approx \mathrm{e}^{-1} \times\left[\left|E_{\mathrm{HOMO}}{ }^{\text {donor }}\right|-\right.$ $\left.\left|E_{\mathrm{LUMO}}{ }^{\text {acceptor }}\right|\right]$, 结合 $\mathrm{P} 3 \mathrm{HT}$ 的 HOMO 能级, 可以确定, 其 与 $\mathrm{P} 3 \mathrm{HT}$ 制备的电池的开路电压将会比 $\mathrm{P} 3 \mathrm{HT} / \mathrm{PC}_{61} \mathrm{BM}$ 电池明显提高.
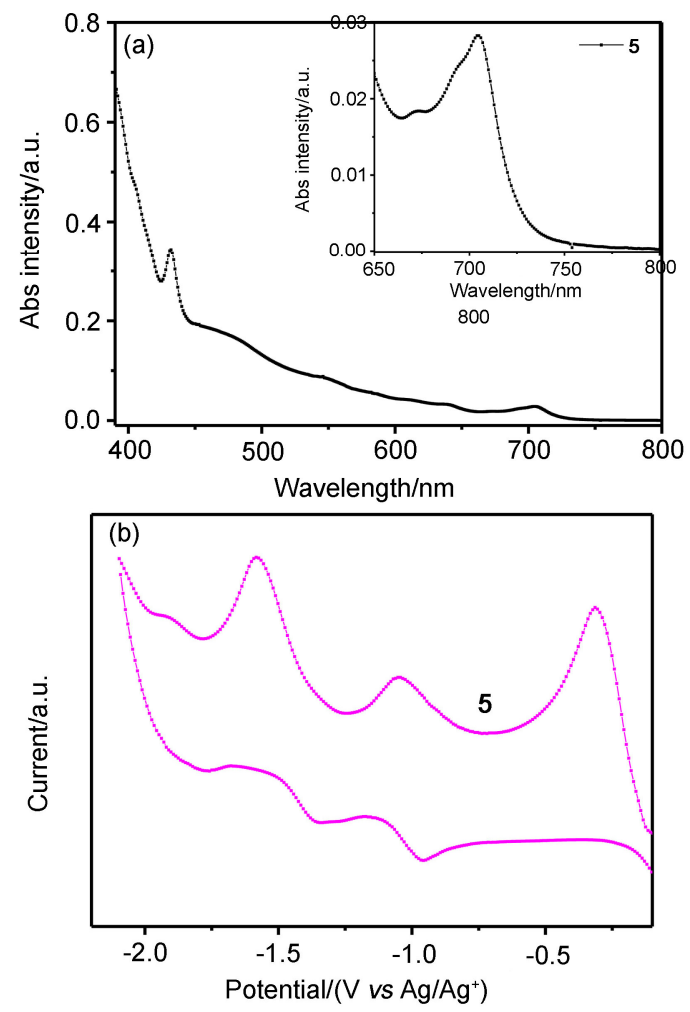

图 1 (a)化合物 $\mathbf{5}$ 的紫外吸收光谱和(b)目标化合物的电化学 性质

Figure 1 (a) The UV-vis absorption spectra of compound 5 and (b) the electrochemical properties of compound $\mathbf{5}$ and $\mathrm{C}_{60}$ Insert: the enlarged view in the wavelength of $708 \mathrm{~nm}$

表 1 双富勒烯衍生物 5 的光物理光化学性能

Table 1 The photophysical and photochemical properties of compound $\mathbf{5}$

\begin{tabular}{ccccc}
\hline Compd. & $\lambda_{\text {abs }} / \mathrm{nm}$ & $\mathrm{LUMO} / \mathrm{eV}$ & $\mathrm{HOMO}^{b} / \mathrm{eV}$ & $E_{\mathrm{g}}{ }^{a} \mathrm{eV}$ \\
\hline $\mathbf{5}$ & 432,708 & -3.68 & -5.39 & 1.71 \\
$\mathrm{PC}_{60} \mathrm{BM}^{c}$ & 431,295 & -3.91 & -5.64 & 1.73 \\
\hline
\end{tabular}

${ }^{a}$ The energy gap of compound $\mathbf{5}$ was calculated from the onset of absoption. ${ }^{b}$ The energy level of HOMO was gotten from the level of LUMO and energy gap $\left(E_{\mathrm{g}}\right){ }^{c}$ The values are referred from the publication of J. Am. Chem. Soc. (2010, 132, 1377). 


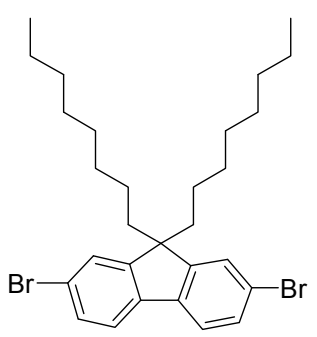

1

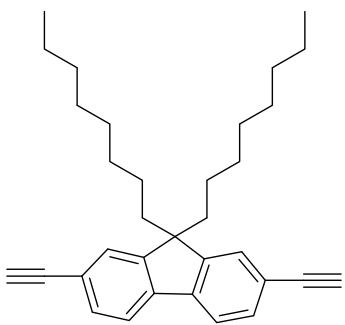

3

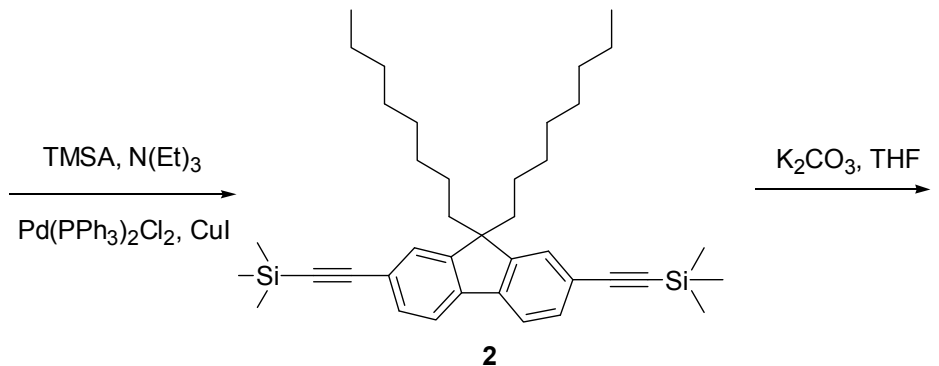

2

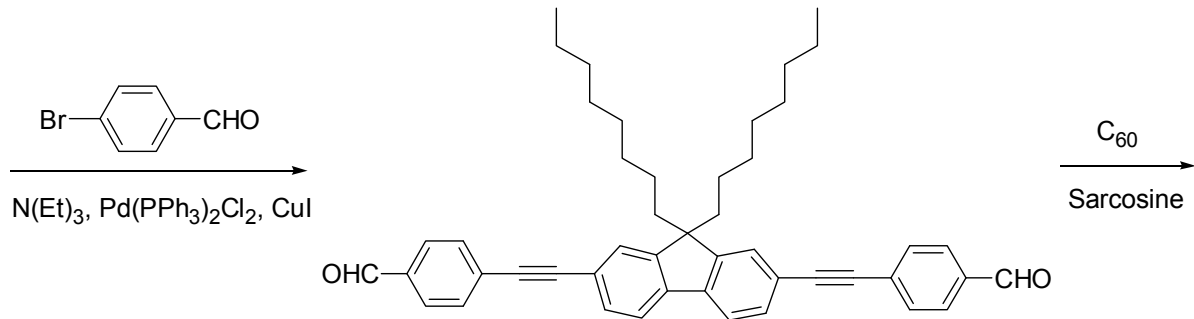

4

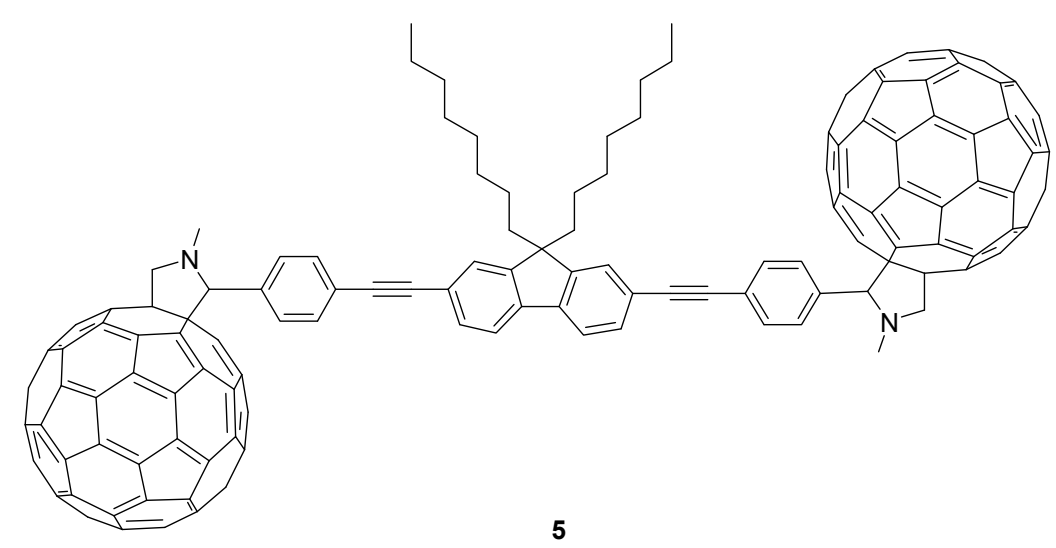

图式 1 含双富勒烯衍生物的合成路线

Scheme 1 The synthetic route of bifullerene derivative

\section{2 理论计算}

目标化合物的分子结构采用 Gaussian 03 程序 ${ }^{[18]}$ 中 的标准 B3LYP/3-21G*方法对其进行优化计算, 分子结 构首先采用 Hyperchem 中的 AM1 方法对其进行初步优 化, 然后使用高斯 03 程序对其进行优化, 优化后的几何 构型如下图 2 所示.

计算结果发现 $\mathrm{C}_{60}$ 与中间连接基团最近距离 $(\mathrm{Rcc})$ 约 为 $0.9 \mathrm{~nm}$, 此结果将有利于分子内和分子间电荷转移态 的形成, 而聚合物太阳能电池通过光吸收后直接产生分 子间的电荷转移过程, 因此, 此设计合成的双富勒烯衍 生物将有利于作为聚合物太阳能电池的受体材料. 而 且, 富勒烯球上的键长 $r_{56-57}$ 功能化后明显增加, 这是由 于其键的杂化形式从 $\mathrm{sp}^{2}$ 变 $\mathrm{sp}^{3}$ 所致.

\section{3 聚合物太阳能电池性能}

制备结构为 ITO/PEDOT:PSS/P3HT:5/Ca/Al 的异质 结太阳能电池, 为了获得最佳的电池性能, 活性层材料
的厚度尤为关键, 通过对活性层的浓度进行调节, P3HT 和化合物 5 的比例从质量比为 $1: 0.5$ 调整到 $1: 1.5$, 研 究结果表明 P3HT 和化合物 5 的最佳配比为为 $1: 0.5$, 其器件的电流-电压曲线、光伏数据列在表 2 中. 从表 2 中可以发现, 当 P3HT 与化合物 5 的质量比为 $1: 0.5$ 时, 其开路电压为 $0.89 \mathrm{~V}$, 电流为 $1.52 \mathrm{~mA} / \mathrm{cm}^{2}$; 而通过改变 化合物 $\mathbf{5}$ 的浓度, 其开路电压始终保持在 $0.86 \mathrm{~V}$ 至 0.91 $\mathrm{V}$ 之间.

表 2 不同给受体比例太阳能电池光伏性能

Table 2 The photovoltaic properties of PSCs based on different ratio of $\mathrm{P} 3 \mathrm{HT}$ and compound $\mathbf{5}$

\begin{tabular}{ccccc}
\hline P3HT $: \mathbf{5}$ & $V_{\mathrm{oc}} / \mathrm{V}$ & $J_{\mathrm{sc}} /\left(\mathrm{mA} \cdot \mathrm{cm}^{-2}\right)$ & $\mathrm{FF} / \%$ & $\mathrm{PCE} / \%$ \\
\hline $1: 0.5$ & 0.89 & 1.52 & 36.2 & 0.56 \\
$1: 1.0$ & 0.91 & 1.19 & 38.2 & 0.33 \\
$1: 1.5$ & 0.86 & 0.99 & 40.2 & 0.21 \\
\hline
\end{tabular}




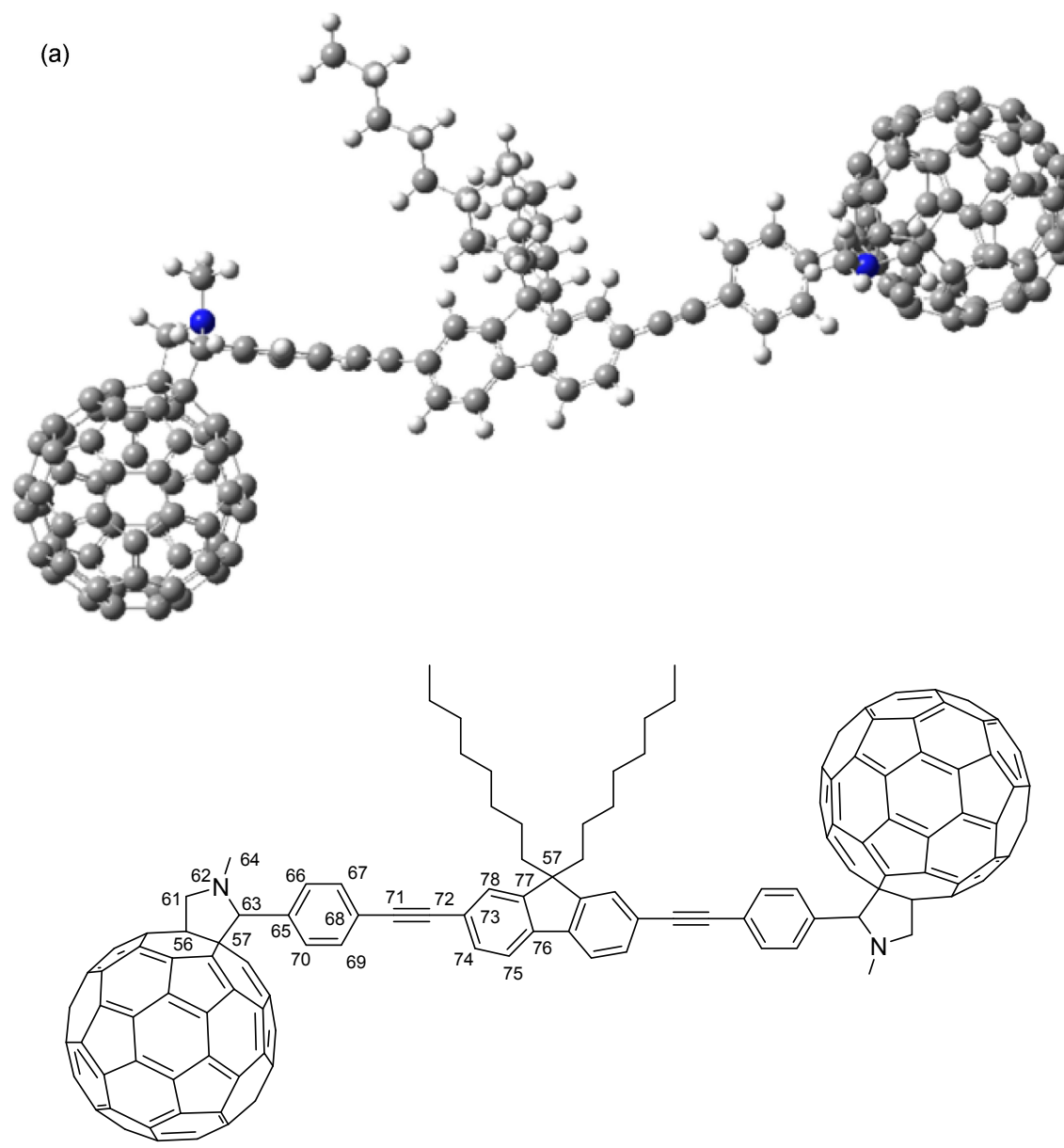

图 2 (a)目标化合物的最优几何构象图和(b)分子中对应的原子序数

Figure 2 (a) The optimized geometry and (b) partly atomic numbers of target molecule

为了进一步优化器件性能, 在实验中通过调节活性 层的厚度, 主要是通过改变活性层的旋涂速率来调整活 性层的厚度, 其结果列在表 3 中. 从表中可以看出, 当 旋速为 $1300 \mathrm{r} / \mathrm{min}$ 时, 其器件表现出最好的结果. 鉴于 此, 后续的活性层成膜均采用此旋涂速率进行旋涂. 接 着尝试使用添加剂来提高器件的性能, 结果发现加入少 量 1,8-二碘辛烷(DIO)或 1-氯荎(1-CN)后, 器件性能出现 明显降低(表3), 图 3 是电池活性层形貌的原子力显微镜

表 3 不同旋涂速率和添加剂下的太阳能电池光伏性能

Table 3 The photovoltaic properties of PSCs based on different annealing temperatures

\begin{tabular}{ccccccc}
\hline $\begin{array}{c}\text { Spin coating } \\
\text { speed/ } \\
\left(\mathrm{r} \bullet \mathrm{min}^{-1}\right)\end{array}$ & $\begin{array}{c}\text { Thickness/ } \\
\mathrm{nm}\end{array}$ & Additives $V_{\mathrm{oc}} / \mathrm{V}$ & $\begin{array}{c}J_{\text {sc }} / \\
\left(\mathrm{mA} \bullet \mathrm{cm}^{-2}\right)\end{array}$ & $\mathrm{FF} / \% \mathrm{PCE} / \%$ \\
1000 & 140 & - & 0.84 & 2.15 & 24.4 & 0.46 \\
1300 & 110 & - & 0.89 & 1.75 & 37.7 & 0.79 \\
1300 & 110 & $\begin{array}{c}\mathrm{DIO} \\
(1 \mathrm{wt} \%)\end{array}$ & 0.87 & 1.26 & 35.3 & 0.61 \\
1300 & 110 & $\begin{array}{c}1-\mathrm{CN} \\
(1 \mathrm{wt} \%)\end{array}$ & 0.87 & 1.32 & 28.4 & 0.24 \\
1500 & 80 & - & 0.88 & 1.06 & 38.6 & 0.36 \\
\hline
\end{tabular}

(AFM)图, 从图中可以看出, $\mathrm{a}, \mathrm{b}, \mathrm{c}$ 三图均出现不同程 度的聚集, 尤其是 $\mathrm{b}$ 和 $\mathrm{c}$ 两图, 相比 $\mathrm{a}$ 图, $\mathrm{b}$ 和 $\mathrm{c}$ 图的表 面形貌更为粗粗, 开始出现相分离现象, 其明显不利于 电荷的传导, 导致了器件 PCE 较低, 这与相应器件的结 果也是一致的.

因而, 接下来的器件制作中均未添加任何添加剂. 最后, 采用不同温度下对器件的活性层进行退火处理, 图 $4 \mathrm{~b}$ 展示了 P3HT 和化合物 5 在 $90 \sim 120{ }^{\circ} \mathrm{C}$ 下退火 15 $\min$ 的电流-电压曲线, 器件性能参数列在表 3 中. 我们 发现 $110{ }^{\circ} \mathrm{C}$ 退火 $15 \mathrm{~min}$ 得到的器件光电转换效率( $\mathrm{PCE}$ ) 最高, 达到 $1.01 \%$, 其开路电压 $\left(V_{\mathrm{oc}}\right)$ 为 $0.89 \mathrm{~V}$, 短路电流 $\left(J_{\mathrm{sc}}\right)$ 为 $2.73 \mathrm{~mA} / \mathrm{cm}^{2}$, 填充因子 $(\mathrm{FF})$ 为 $41.2 \%$. 当退火温 度进一步提高, 结果发现电池的效率出现明显的下降, 尤其是 $J_{\mathrm{sc}}$ 的降低. 这一结果表明, 当退火温度选择适 中, 可能有利于活性层中形成更好的空间网络互穿结 构, 这样将有利于载流子的分离与和传输, 从而提高器 件的光电转换效率. 为了便于比较, 同样, 我们也制作 了基于 $\mathrm{P} 3 \mathrm{HT}: \mathrm{PC}_{60} \mathrm{BM}$ 作为活性层的相同结构器件, 其 器件的开路电压为 $0.55 \mathrm{~V}$, 电流密度为 $8.82 \mathrm{~mA} / \mathrm{cm}^{2}$, 

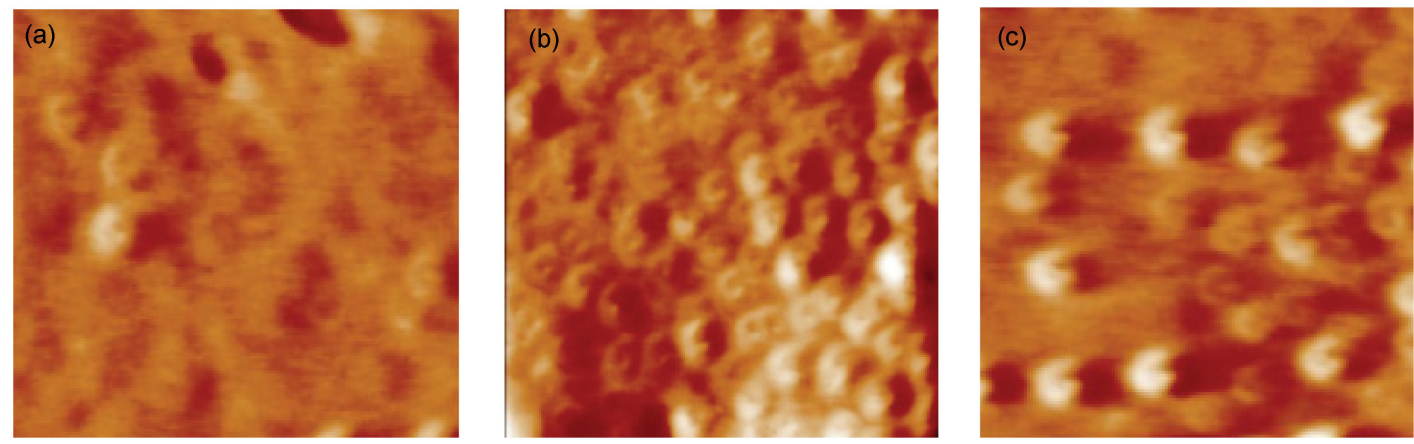

图 3 基于 P3HT 与化合物 $\mathbf{5}$ 的电池活性层的 AFM 的表面形貌图

Figure 3 The morphology of active-layer based on P3HT and compound 5

(a) No additive; (b) $1 \% 1-\mathrm{CN}$; (c) $1 \% \mathrm{DIO}$

表 4 有利于活性层中形成更好的空间网络互穿结构 ${ }^{a}$

Table 4 The photovoltaic properties of PSCs based on different annealing temperatures

\begin{tabular}{|c|c|c|c|c|c|}
\hline Annealing temperature $/{ }^{\circ} \mathrm{C}$ & P3HT : 5 & $V_{\mathrm{oc}} / \mathrm{V}$ & $J_{\mathrm{sc}} /\left(\mathrm{mA} \cdot \mathrm{cm}^{-2}\right)$ & $\mathrm{FF} / \%$ & $\mathrm{PCE} / \%$ \\
\hline 90 & $1: 0.5$ & $0.87 \pm 0.02$ & $1.79 \pm 0.01$ & $38.8 \pm 0.02$ & $0.84 \pm 0.01$ \\
\hline 110 & $1: 0.5$ & $0.89 \pm 0.02$ & $2.73 \pm 0.03$ & $39.1 \pm 0.02$ & $1.01 \pm 0.02$ \\
\hline 120 & $1: 0.5$ & $0.90 \pm 0.02$ & $1.49 \pm 0.01$ & $37.6 \pm 0.02$ & $0.46 \pm 0.01$ \\
\hline P3HT:PC ${ }_{61} \mathrm{BM}$ & $1: 1.0$ & $0.55 \pm 0.01$ & $8.82 \pm 0.02$ & $58.2 \pm 0.02$ & $2.68 \pm 0.02$ \\
\hline
\end{tabular}

测量结果为 20 个样品的算术平均值加上偏差.
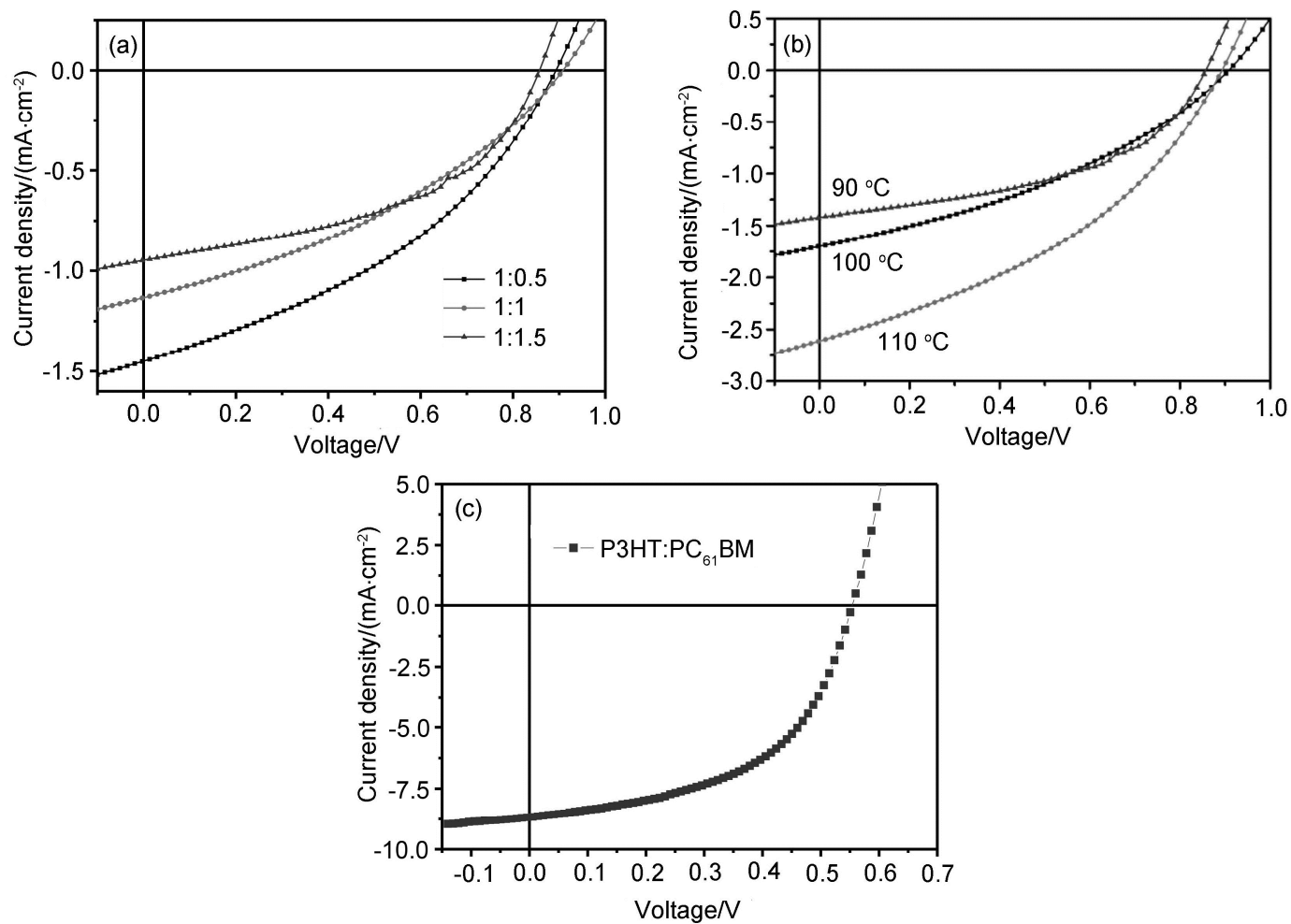

图 4 基于 P3HT:5 的(a)不同质量比、(b)不同退火温度下以及 P3HT:PC61BM.的太阳能电池电流-电压曲线

Figure 4 The curves of current density vs voltage in these PSCs with (a) different mass ratio of P3HT:5, (b) different annealing of P3HT:5 and (c) P3HT:PC61BM

填充因子为 $58.2 \%$, 器件的光电转换效率为 $2.68 \%$. 从 表 4 和 P3HT:PC 60 BM 型器件可以看出, 器件的开路电压
提高了 $\approx 0.34 \mathrm{~V}$, 而电流密度远低于 $\mathrm{P} 3 \mathrm{HT}: \mathrm{PC}_{60} \mathrm{BM}$ 型器 件, 这主要是由于 P3HT:5 混合膜出现明显的聚集现象, 
没有形成有效的空间网络互穿结构, 吸收光产生的空穴 和电子不能够传出, 从而严重的影响了其器件的短路电 流.

\section{2 结论}

本文设计合成了具有双富勒烯结构单元的富勒烯 衍生物, 通过对其结构的设计, 有效地改变其在有机溶 剂中的溶解性, 将其与聚合物 P3HT 共混后制备太阳能 电池, 当 P3HT 和化合物 5 的质量混合比为 $1: 0.5$, 退 火温度为 $110{ }^{\circ} \mathrm{C}$ 时, 其太阳能电池开路电压达到了 0.89 $\mathrm{V}$, 光电转换效率为 $1.01 \%$, 短路电流为 $2.73 \mathrm{~mA} / \mathrm{cm}^{2}$, 填充因子 $\mathrm{FF}$ 为 $39.1 \%$, 此设计为获得高开路电压的聚合 物太阳能电池提高一种可行性选择. 鉴于此, 为了获得 高效率基于杜铃状双富勒烯太阳能电池, 一种行之有效 的方法是通过与 $\mathrm{PC}_{60} \mathrm{BM}$ 作为共同受体，从而实现其高 的光电转换效率.

\section{3 实验部分}

\section{1 仪器与试剂}

DRX-400 MHz 核磁共振仪 (TMS 为内标, $\mathrm{CDCl}_{3}$ 为 溶剂, 德国 Bruker 公司); 气相色谱一质谱仪(直接进样, 美国 FINIGAN 公司)和基质辅助激光解吸电离飞行时间 质谱(直接进样, 德国 Bruker 公司); 元素分析(仪美国 FINIGAN 公司); 紫外吸收和荧光发射光谱仪 (日本 HITACHI 公司).

2,7-二溴-9,9-二辛基芴(1)、三甲基硅乙炔(TMSA)、 三乙胺(TEA)、二氯(二三苯基膦)钯 $\left[\mathrm{Pd}\left(\mathrm{PPh}_{3}\right)_{2} \mathrm{Cl}_{2}\right]$ 、对溴 苯甲醛、富勒烯 $\mathrm{C}_{60}$ 均购自百灵威化学试剂公司, 柱层 析硅胶使用青岛海洋化工厂的(100 200 目). 其它试剂 均为市售分析纯购自国药试剂公司, 除特殊说明, 未经 处理直接使用.

\section{2 化合物 2 的合成}

化合物 2 的合成根据参考文献[19, 20], 产率 72\%.

\section{3 化合物 3 的合成}

化合物 3 的合成根据参考文献[19, 20], 产率 95\%. 化合物 3 没有进行进一步纯化，直接进行下一步反应.

\section{4 化合物 4 的合成}

将 $2.96 \mathrm{~g}$ 的对溴苯甲醛 $(16 \mathrm{mmol}), 3.07 \mathrm{~g}$ 的化合物 3 (7.2 mmol), $0.264 \mathrm{~g} \mathrm{Pd}\left(\mathrm{PPh}_{3}\right)_{2} \mathrm{Cl}_{2}(0.376 \mathrm{mmol}), 0.136 \mathrm{~g}$ 的 碘化亚铜 $(0.72 \mathrm{mmol})$ 加入到 $250 \mathrm{~mL}$ 三口烧瓶中, 氞气 保护, 加入 $120 \mathrm{~mL}$ 除水的三乙胺, 升度至 $50{ }^{\circ} \mathrm{C}$, 摚拌 过夜. 反应结束后, 蒸干溶剂, 将混和物溶于 $30 \mathrm{~mL}$ 的 二氯甲烷, 过滤, 滤液使用梯度洗脱的方法进行柱色谱 分离纯化, 洗脱剂为二氯甲烷和石油醚. 纯产物在真空
干燥得到 $2.42 \mathrm{~g}$ 的黄色粉末化合物 4, 产率 75\%. m.p. $254 \sim 255{ }^{\circ} \mathrm{C} ;{ }^{1} \mathrm{H}$ NMR $\left(\mathrm{CDCl}_{3}, 400 \mathrm{MHz}\right) \delta: 10.03$ (s, $2 \mathrm{H}), 7.89(\mathrm{~d}, J=8.0 \mathrm{~Hz}, 6 \mathrm{H}), 7.71(\mathrm{~d}, J=8.2 \mathrm{~Hz}, 4 \mathrm{H})$, $7.57 \sim 7.54(\mathrm{~m}, 4 \mathrm{H}), 2.02 \sim 1.98(\mathrm{~m}, 4 \mathrm{H}), 1.25 \sim 1.06(\mathrm{~m}$, 24), 0.81 (t, $J=6.8 \mathrm{~Hz}, 6 \mathrm{H})$; MS (EI) $m / z(\%): 646.1\left(\mathrm{M}^{+}\right)$. Anal. calcd for $\mathrm{C}_{47} \mathrm{H}_{50} \mathrm{O}_{2} \mathrm{C}$ 87.26, $\mathrm{H}$ 7.79; found $\mathrm{C}$ 87.35.98, H 7.83.

\section{5 化合物 5 的合成}

称取 $1.5 \mathrm{~g} \mathrm{C}_{60}(2.3 \mathrm{mmol}), 3.29 \mathrm{~g}$ 肌氨酸 $(37 \mathrm{mmol})$ 和 $0.323 \mathrm{~g}$ 化合物 $4(0.5 \mathrm{mmol})$ 于 $250 \mathrm{~mL}$ 三口烧瓶中, 加 入 $120 \mathrm{~mL}$ 甲苯, 在氮气保护下加热回流, TLC 监控. 12 $\mathrm{h}$ 后停止反应, 冷却至室温, 得到红棕色液体, 减压蒸 去溶剂后以甲苯/环己烷作为洗脱剂梯度洗脱分离, 得 到棕色固体 $0.31 \mathrm{~g}$, 产率 $38.2 \%$. m.p. $>300{ }^{\circ} \mathrm{C} ;{ }^{1} \mathrm{H}$ NMR $\left(\mathrm{CDCl}_{3}, 400 \mathrm{MHz}\right) \delta: 7.84 \sim 7.79(\mathrm{~m}, 4 \mathrm{H}), 7.60$ (t, $J=7.8$ $\mathrm{Hz}, 6 \mathrm{H}), 7.44$ (t, $J=8.2 \mathrm{~Hz}, 4 \mathrm{H}), 5.0(\mathrm{~d}, J=8.0 \mathrm{~Hz}, 2 \mathrm{H})$, $4.98(\mathrm{~s}, 2 \mathrm{H}), 4.30(\mathrm{~d}, J=8.8 \mathrm{~Hz}, 2 \mathrm{H}), 2.85(\mathrm{~s}, 6 \mathrm{H}), 1.97 \sim$ $1.93(\mathrm{~m}, 4 \mathrm{H}), 1.37 \sim 1.24(\mathrm{~m}, 10 \mathrm{H}), 1.09 \sim 1.01(\mathrm{~m}, 14)$, $0.83(\mathrm{t}, J=7.2 \mathrm{~Hz}, 6 \mathrm{H}) ;{ }^{13} \mathrm{C} \mathrm{NMR}\left(\mathrm{CDCl}_{3} / \mathrm{CS}_{2}, 100 \mathrm{MHz}\right)$ $\delta: 155.9,153.6,152.9,152.7,150.8,147.1,146.4,146.2$, $146.1,146.0,145.9,145.8,145.7,147.6,145.5,145.4$, $145.3,145.2,145.1,145.0,144.9,144.8,144.7,144.6$, $144.4,144.3,144.1,143.0,142.9,142.5,142.4,142.3$, $142.2,142.1,142.0,141.9,141.8,141.7,141.6,141.5$, $145.4,141.3,141.1,140.2,140.0,139.9,139.8,139.4$, $136.9,136.7,136.2,135.7,135.5,135.1,131.7,130.6$, $130.1,129.1,127.8,125.8,123.5,121.8,119.7,91.3,89.8$, $83.1,69.9,68.8,54.9,39.8,31.8,30.1,29.3,24.7$, 23.8,22.7, 14.1; MALDI-TOF-MS $m / z(\%): 2140.12\left(\mathrm{M}^{+}\right)$. Anal. calcd for $\mathrm{C}_{171} \mathrm{H}_{60} \mathrm{~N}_{2} \mathrm{C}$ 95.89, H 2.80, N 1.31; found C 95.36, H 2.91, N 1.73 .

\section{6 器件的制作与测试}

基于新颖含双富勒烯结构单元的衍生物作为受体 材料的太阳能电池的结构为: ITO/PEDOT:PSS/P3HT:富 勒烯衍生物/Ca/Al. 器件制作过程中 ITO 基底清洗是分 别依次采用去离子水、丙酮、异丙醇超声波清洗 $30 \mathrm{~min}$, 然后通过氮气枪吹燥，再将过滤后的 PEDOT:PSS 旋涂 于 ITO 基底上, 旋涂速度为 $4000 \mathrm{r} / \mathrm{min}$, 在 $120{ }^{\circ} \mathrm{C}$ 下退 火; 聚三己基噻吩(P3HT) 和富勒烯衍生物以不同的质量 比溶于氯仿和邻二氯苯的混合溶剂, 加热搅拌溶解, 在 手套箱中通过旋涂成膜于 PEDOT:PSS 上，选择不同的 退火温度进行退火处理, 然后再蒸镀金属钙和电极铝, 其厚度分别为 20 和 $100 \mathrm{~nm}$, 电池有效面积为 $0.08 \mathrm{~cm}^{2}$; 电池的光电转换效率则是通过模拟太阳光强 (1.5 AM) 的 条件下, 通过 Keithly 2400 数字源表测定器件的电流-电 
压.

\section{7 电化学测试}

电化学测试是在上海辰华公司 CHI660e 仪器上完 成的, 测试过程中采用三电极系统, 以玻碳电极作为工 作电极, 铂丝作为对电极, $\mathrm{Ag} / \mathrm{Ag}^{+}$作为参比电极, 电解 质溶液为 $0.1 \mathrm{moL} / \mathrm{L}$ 的 $\mathrm{Bu}_{4} \mathrm{NPF}_{6}$ 的乙腈溶液. 参比电极 采用用 $\mathrm{Fc} / \mathrm{Fc}^{+}$进行校准. LUMO 根据参考文献[21]中所 涉及的公式计算得出, 其中 $4.80 \mathrm{eV}$ 是二茂铁在真空下 的能级.

\section{References}

[1] Chen, L. M.; Hong, Z. R.; Li, G.; Yang, Y. Adv. Mater. 2009, 21, 1434

[2] Qin, R. P.; Song, G. L.; Jaing, Y. R.; Bo, Z. S. Chem. J. Chin. Univ. 2012, 33, 828 (in Chinese).

(秦瑞平, 宋桂林, 蒋玉荣, 薄志山, 高等学校化学学报, 2012, 33, 828.)

[3] Yao, J. B.; Wang, Y. H.; Zhang, H. Z. Chem. Res. Chin. Univ. 2013, 29, 1185.

[4] Sariciftci, N. S.; Smilowitz, L.; Heeger, A. J. Science 1992, 258, 1474.

[5] Zhao, H. Y.; Guo, X. Y.; Tian, H. K.; Li, C. Y.; Xie, Z. Y.; Geng, Y. H.; Wang, F. S. J. Mater. Chem. 2010, 20, 3092.

[6] Choi, J. H.; Son, K. I.; Kim, T.; Kim, K.; Ohkubo, K.; Fukuzumi, S. J. Mater. Chem. 2010, 20, 475.

[7] Chang, C. L.; Liang, C. W.; Syu, J. J.; Wang, L.; Leung, M. K. Sol. Energy Mater. Sol. Cells 2011, 95, 2371.

[8] Deigado, J. L.; Martin, N.; de la Cruz P.; Langa, F. Chem. Soc. Rev. 2011, 40, 5232.

[9] Backer, S. A.; Sivula, K.; Kavulak, D. F.; Fréchet, J. M. J. Chem. Mater. 2007, 19, 2927.
[10] Lenes, M.; Wetzelaer, G. J. A. H.; Kooistra, F. B.; Veenstra, S. C.; Hummelen, J. C.; Blom, P. W. M. Adv. Mater. 2008, 20, 2116.

[11] Zhao, Y.; Zhang, F. L.; Zhang, M.; Ma, Y. G. Chem. Res. Chin. Univ. 2013, 29, 1193.

[12] Zhang, C. Y.; Chen, S. C.; Xiao, Z.; Zuo, Q. Q.; Ding, L M. Org. Lett. 2012, 14, 1508.

[13] He, D.; Du, X. Y.; Xiao, Z.; Ding, L. M. Org. Lett. 2014, 16, 612.

[14] Sui, N.; Huang, T. H.; Wang, Y. H.; Zhang, H. Z. Chem. Res. Chin. Univ. 2014, 30, 513.

[15] (a) He, Y. J.; Zhao, G. J.; Peng, B.; Li, Y. F. Adv. Funct. Mater. 2010, 20, 3383.

(b) Zhao, G. J.; He, Y. J.; Li, Y. F. Adv. Mater. 2010, 22, 4355.

[16] Wong, W.; Vak, W. H.; Singh, D.; Ren, T. B.; Yan, S. J.; Jones, C.; Liaw, D. J.; Lamb,I. I.; Holmes, R. N.; Org. Lett. 2010, 12, 5000.

[17] Lu, J. P.; Ding, J. F.; Alem, S.; Waklm, S.; Tse, S. C.; Tao, Y.; Stupak, J.; Li, J. J. J. Mater. Chem. 2011, 21, 4953

[18] Frisch, M. J.; Trucks, G. W.; Schlegel, H. B.; Scuseria, G. E.; Robb, M. A.; Cheeseman, J. R.; Zakrzewski, V. G.; Montgomery, J. A.; Stratmann, R. E.; Burant, J. C.; Dapprich, S.; Daniels, A. D.; Kudin, K. N.; Strain, M. C.; Farkas, O.; Tomasi, J.; Barone, V.; Cossi, M.; Cammi, R.; Mennucci, B.; Pomelli, C.; Adamo, C.; Clifford, S.; Ochterski, J.; Peterson, G. A.; Ayala, P. Y.; Cui, Q.; Morokuma, K.; Malik, D. K.; Rabuk, A. D.; Raghavachari, K.; Foresman, J. B.; Cioslowski, J.; Ortiz, J. V.; Stefanov, B. B.; Liu, G.; Liashenko, A.; Piskorz, P.; Komaromi, I.; Gomperts, R.; Martin, R. L.; Fox, D. J.; Keith, T.; Al-Laham, M. A.; Peng, C. Y.; Nanayakkara, A.; Gonzalez, C.; Chalacombe, M.; Gill, P. M. W.; Johnson, B. G.; Chen, W.; Wong, M. W.; Andres, J. L.; Head-Gordon, M.; Replogle, E. S.; Pople, J. A. GAUSSIAN 2003, Gaussian, Inc., Pittsburgh, PA, 2003.

[19] Liu, S. J.; Zhang, K.; Lu, J. M.; Zhang, J.; Yip, H.-L.; Cao, Y. J. Am. Chem. Soc. 2013, 135, 15326

[20] Maji, M. S.; Pfeifer, T.; Studer, A. Chem.-Eur. J. 2010, 16, 5872

[21] Ouyang, X. H.; Chen, D. C.; Zeng, S. M.; Zhang, X. Y.; Su, S. J.; Ge, Z. Y. J. Mater. Chem. 2012, 22, 23005 